\title{
Topological transitions in arrays of dipoles coupled to a cavity waveguide
}

\author{
Charlie-Ray Mann $~_{*}^{*}$ and Eros Mariani $~^{\dagger}{ }^{\dagger}$ \\ School of Physics and Astronomy, University of Exeter, Exeter EX4 4QL, United Kingdom.
}

(Received 26 October 2020; accepted 9 November 2021; published 1 February 2022)

\begin{abstract}
Topological valley-Hall edge states have been realized in a variety of photonic structures across the electromagnetic spectrum because they can be easily engineered by breaking certain lattice symmetries. However, the valley-Chern numbers that characterize the topological phase are usually fixed by the symmetry-breaking perturbation and therefore the valley-Hall edge states are forced to propagate in a fixed direction. Here we consider a kagome metasurface comprised of a subwavelength array of dipole emitters/antennas, and we unveil that one can modify the geometrical and topological properties of the polaritons by structuring the local photonic environment. As a proof of concept, we show that one can induce topological transitions via accidental Dirac points by embedding the metasurface inside a cavity waveguide. Varying the cavity width modifies the nature of the dipole-dipole interactions which enables one to manipulate the Berry curvature and invert the valley-Chern numbers without inverting the symmetry-breaking perturbation. Consequently, we demonstrate that one can switch the chirality of the polariton valley-Hall edge states by varying only the cavity width. This alternative approach to engineering topological transitions via structuring the photonic environment could also have implications for other topological phases such as photonic higher-order topological insulators.
\end{abstract}

DOI: 10.1103/PhysRevResearch.4.013078

\section{INTRODUCTION}

Topological phases of light exhibit unique properties beyond the realm of conventional photonics [1-15]. Within this paradigm, the valley-Hall insulator has attracted considerable interest because it does not require one to break time-reversal symmetry; instead, the valley-Hall phase is easily induced by breaking certain lattice symmetries [15]. Consequently, valley-Hall insulators have been successfully realized in a variety of photonic structures at microwave [16-18], terahertz [19,20], and optical [21-25] frequencies.

These works begin with hexagonal lattices that exhibit deterministic Dirac points and then engineer a mass gap by breaking the inversion and/or mirror symmetries. This generates localized Berry curvature near the inequivalent valleys and the corresponding phase is characterized by nontrivial valley-Chern numbers. While these are not strictly topological invariants, there still exists a bulk-boundary correspondence [26-33]. An interface separating two regions with opposite valley-Chern numbers support valley-polarized chiral edge states which can be exploited to transport light through sharp bends and certain disorder that does not strongly mix the valleys [16-25] - a tantalizing prospect that could have crucial implications for future photonic devices.

\footnotetext{
*cm433@exeter.ac.uk

†E.Mariani@exeter.ac.uk
}

Published by the American Physical Society under the terms of the Creative Commons Attribution 4.0 International license. Further distribution of this work must maintain attribution to the author(s) and the published article's title, journal citation, and DOI.
However, this simple geometrical origin of the valley-Hall insulator usually comes at a price: The topological phase is typically fixed by the symmetry-breaking perturbation that is imprinted into the lattice design. To deterministically change the valley-Chern numbers one needs to invert the symmetry-breaking perturbation to induce a topological transition, passing through a critical point where the symmetry that gives rise to the deterministic Dirac points is restored. However, it is usually difficult to reconfigure every unit cell in the photonic lattice after it has been fabricated. Consequently, the valley-Chern numbers are generally fixed by design and therefore the topological edge states are forced to propagate in a fixed direction. This raises an intriguing question: Is it possible to induce a topological transition without inverting the symmetry-breaking perturbation?

To address this question, here we theoretically consider a kagome metasurface that is comprised of a subwavelength array of resonant dipoles, which could be realized with classical antennas or quantum emitters. The metasurface hybridizes with the surrounding photons which give rise to polaritons whose spectrum exhibits deterministic Dirac points due to the underlying symmetries. To induce the valley-Hall phase we generate a mass gap by perturbing the unit cell which breaks the inversion and mirror symmetries. Crucially, given the hybrid light-matter nature of the polaritons, the Berry curvature and corresponding valley-Chern numbers are not immutable characteristics that are fixed by the symmetry-breaking perturbation; they also depend qualitatively on the local photonic environment which mediates the dipole-dipole interactions. This opens the possibility of engineering topological transitions by structuring the local photonic environment.

As a proof of concept, we consider a minimal model of point dipole emitters/antennas coupled to a cavity waveguide. 
(a)
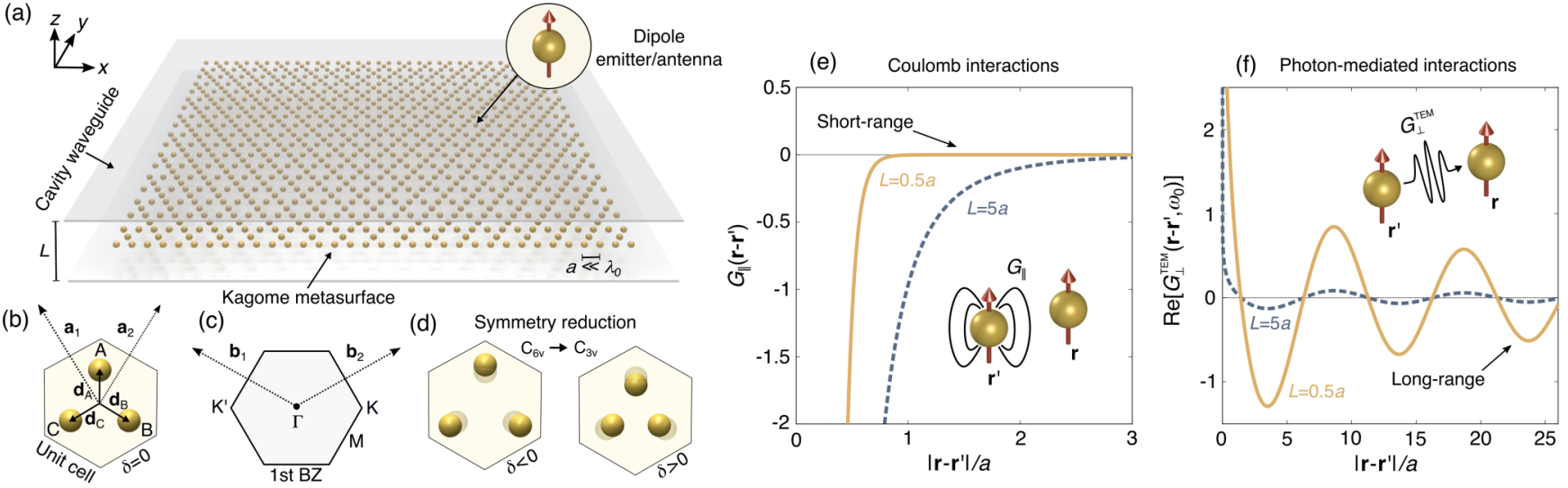

FIG. 1. (a) Schematic of a kagome metasurface comprised of an array of dipole emitters/antennas with subwavelength nearest-neighbor separation $a \ll \lambda_{0}$. The induced dipole moments are assumed to point in the $z$ direction (see inset). Furthermore, the metasurface is embedded inside a cavity waveguide of width $L$, where the cavity walls are assumed to be perfect mirrors. (b) Corresponding unit cell and (c) first Brillouin zone. (d) Expanding (left) or shrinking (right) the distance between the dipoles in the unit cell reduces the point group of the metasurface from $C_{6 v}$ to $C_{3 v}$ and the little point group of the $\mathrm{K} / \mathrm{K}^{\prime}$ points from $C_{3 v}$ to $C_{3}$. (e) Longitudinal Green's function which describes the short-range Coulomb interactions for $L=5 a$ (dashed blue line) and $L=0.5 a$ (solid orange line). (f) Real part of the transverse Green's function which describes the coherent long-range interactions mediated by the TEM cavity photons for $L=5 a$ (dashed blue line) and $L=0.5 a$ (solid orange line). Varying the cavity width enables one to modify the dominant dipole-dipole coupling mechanism. Results obtained with $a=0.1 \lambda_{0}$.

For the transverse excitations, one can modify the dominant dipolar coupling from short-range Coulomb interactions to long-range photon-mediated interactions by varying the cavity width. Previously, we have shown that this mechanism can induce exotic Dirac point merging transitions in a honeycomb metasurface, which enables one to manipulate the properties of type-I and type-II Dirac polaritons while preserving the underlying symmetries [34]. More recently, we have shown that this mechanism enables one to tune the strength of the pseudomagnetic field in a strained metasurface without modifying the strain pattern, which leads to a collapse and revival of the polariton Landau levels [35].

In this work we show that, for a fixed symmetry-breaking perturbation, one can tune the Dirac mass by varying the cavity width; in fact, one can even make the Dirac mass vanish at a critical width without altering the lattice. This results in accidental Dirac points emerging in the polariton spectrum, which signifies a topological transition where the Dirac mass and Berry curvature change sign. Consequently, we demonstrate that one can invert the valley-Chern numbers and thus switch the chirality of the polariton valley-Hall edge states by modifying a single global parameter: The cavity width. While we have focused on a cavity waveguide, we stress that there are other ways to structure the photonic environment which may provide alternative mechanisms of inducing topological transitions.

\section{KAGOME METASURFACE MODEL}

In Fig. 1(a) we schematically depict a kagome metasurface that is composed of an array of polarizable dipole emitters/antennas. We keep the model general because we envisage that the essential physics could be realized in a variety of experimental setups, ranging from classical metasurfaces composed of microwave antennas [34,35] or plasmonic particles [34,36-40] to quantum metasurfaces comprised of excitonic particles $[41,42]$ or atomlike emit- ters [43-50] - although our classical analysis is only relevant in the single-excitation subspace [51]. Consequently, we model the dipoles with a generic bare polarizability of the form

$$
\alpha_{\mathrm{B}}(\omega)=\frac{2 \omega_{0} \mu}{\omega_{0}^{2}-\omega^{2}-i \omega \gamma_{\mathrm{nr}}},
$$

where $\omega_{0}$ is the free space resonant frequency, $\mu$ characterizes the strength of the polarizability, and $\gamma_{\mathrm{nr}}$ accounts for nonradiative losses. Furthermore, we restrict our attention to transverse excitations where the dipole moments are oriented in the $z$ direction (see inset).

The kagome metasurface has three inequivalent sublattices which are formed by placing dipoles at periodic positions $\mathbf{R}_{i}=\mathbf{R}+\mathbf{d}_{i}$ where $i=\mathrm{A}, \mathrm{B}, \mathrm{C}$ labels the three sublattices. Here the basis vectors

$$
\begin{aligned}
& \mathbf{d}_{\mathrm{A}}=\frac{a}{\sqrt{3}}[0,1], \quad \mathbf{d}_{\mathrm{B}}=\frac{a}{2 \sqrt{3}}[\sqrt{3},-1], \\
& \mathbf{d}_{\mathrm{C}}=\frac{a}{2 \sqrt{3}}[-\sqrt{3},-1],
\end{aligned}
$$

locate the positions of the three dipoles within each unit cell as depicted in Fig. 1(b). Furthermore, $\mathbf{R}=l_{1} \mathbf{a}_{1}+l_{2} \mathbf{a}_{2}$ represents the set of lattice translation vectors, where $l_{1}, l_{2} \in \mathbb{Z}$ are integers, and

$$
\mathbf{a}_{1}=a[-1, \sqrt{3}], \quad \mathbf{a}_{2}=a[1, \sqrt{3}],
$$

are the primitive lattice vectors. The corresponding set of reciprocal lattice vectors are $\mathbf{g}=n_{1} \mathbf{b}_{1}+n_{2} \mathbf{b}_{2}$, where $n_{1}, n_{2} \in$ $\mathbb{Z}$ are integers, and

$$
\mathbf{b}_{1}=\frac{\pi}{\sqrt{3} a}[-\sqrt{3}, 1], \quad \mathbf{b}_{2}=\frac{\pi}{\sqrt{3} a}[\sqrt{3}, 1],
$$

are the primitive reciprocal lattice vectors that define the Brillouin zone shown in Fig. 1(c). Moreover, we consider the nearest-neighbor separation to be subwavelength $a \ll \lambda_{0}$, where $\lambda_{0}$ is the free space resonant wavelength, so that the 
polaritons near the $\mathrm{K} / \mathrm{K}^{\prime}$ points are subradiant and evanescently bound to the lattice. Finally, we embed the kagome metasurface at the center of a cavity waveguide of width $L$, where the cavity walls are assumed to be perfect mirrors.

\section{A. Symmetry-reducing perturbations}

The point-group symmetry of the kagome metasurface is $C_{6 v}$, while the little point group of the $\mathrm{K} / \mathrm{K}^{\prime}$ points is $C_{3 v}$; consequently, the kagome metasurface can exhibit deterministic Dirac points at the $\mathrm{K} / \mathrm{K}^{\prime}$ points [52]. Note the inversion centers are located at the lattice sites and the corners of the unit cell, while the $C_{3}$ rotation centers are located at the center and corners of the unit cell.

To gap out the Dirac points we introduce the following symmetry-reducing perturbation,

$$
\mathbf{d}_{i} \rightarrow(1-\delta) \mathbf{d}_{i},
$$

where the symmetry-breaking parameter $\delta$ describes the fractional change in the separation distance between the dipoles in the unit cell. Depending on the sign of $\delta$, we have two distinct lattices which we call the shrunken metasurface $(\delta>$ $0)$ and expanded metasurface $(\delta<0)$, and the corresponding unit cells are schematically depicted in Fig. 1(d). We note that a similar strategy has been considered in other artificial kagome lattices [53-59]. This perturbation breaks the inversion symmetry and the mirror symmetry about the $x$ axis, which reduces the point-group symmetry of the metasurface to $C_{3 v}$ and the little point group of the $\mathrm{K} / \mathrm{K}^{\prime}$ valleys to $C_{3}$; consequently, the shrunken and expanded metasurfaces do not exhibit deterministic Dirac points [52].

\section{B. Coupled-dipole equations}

The collective dynamics of the dipoles inside the cavity waveguide are described by a set of coupled-dipole equations

$$
\begin{aligned}
\frac{1}{\alpha(\omega)} p_{\mathbf{R}_{i}}(\omega)= & E_{0}\left(\mathbf{R}_{i}\right)+\sum_{\mathbf{R}_{i}^{\prime} \neq \mathbf{R}_{i}} G\left(\mathbf{R}_{i}-\mathbf{R}_{i}^{\prime}, \omega\right) p_{\mathbf{R}_{i}^{\prime}}(\omega) \\
& +\sum_{j \neq i} \sum_{\mathbf{R}_{j}} G\left(\mathbf{R}_{i}-\mathbf{R}_{j}, \omega\right) p_{\mathbf{R}_{j}}(\omega),
\end{aligned}
$$

where $p_{\mathbf{R}_{i}}$ is the induced dipole moment located at position $\mathbf{R}+\mathbf{d}_{i}$ on the $i$ th sublattice. Furthermore,

$$
\alpha(\omega)=\left[\alpha_{\mathrm{B}}^{-1}(\omega)-\Sigma(\omega)\right]^{-1}
$$

is the renormalized polarizability inside the cavity waveguide where the polarizability correction reads $[35,60]$

$$
\Sigma(\omega)=i \frac{2 a^{3} k_{\omega}^{3}}{3}+\frac{4 a^{3}}{L^{3}}\left[\operatorname{Li}_{3}\left(e^{i k_{\omega} L}\right)-i k_{\omega} L \operatorname{Li}_{2}\left(e^{i k_{\omega} L}\right)\right] .
$$

Here $\operatorname{Li}_{n}(z)=\sum_{t=1}^{\infty} z^{t} / t^{n}$ is the polylogarithm of order $n$, $k_{\omega}=\omega / c$ and $c$ is the speed of light in vacuum. The first term in Eq. (8) is the usual radiative correction in free space while the other terms encode the frequency shifts and modified decay rates that are induced by the cavity waveguide.

Finally, in Eq. (6), $E_{0}(\mathbf{r})$ is the $z$ component of the external driving field, and $G\left(\mathbf{r}-\mathbf{r}^{\prime}, \omega\right)$ is the $z z$ component of the cavity Green's function which reads $[35,60]$

$G\left(\mathbf{r}-\mathbf{r}^{\prime}, \omega\right)=i \frac{\pi a^{3}}{L} \sum_{m=-\infty}^{\infty}\left(k_{\omega}^{2}-k_{m}^{2}\right) H_{0}^{(1)}\left(\sqrt{k_{\omega}^{2}-k_{m}^{2}}\left|\mathbf{r}-\mathbf{r}^{\prime}\right|\right)$.

Here $H_{0}^{(1)}$ is the Hankel function of first kind and zeroth order and $k_{m}=2 m \pi / L$ where $m \in \mathbb{Z}$ is an integer. Physically, this Green's function describes the $z$ component of the field generated at $\mathbf{r}$ by a point dipole source at $\mathbf{r}^{\prime}$ that is oriented along the $z$ direction (both located at the center of the cavity). Therefore the first sum in Eq. (6) encodes the intrasublattice dipole-dipole interactions which are mediated by the cavity waveguide, while the second sum encodes the intersublattice interactions.

\section{Coulomb vs photon-mediated interactions}

The Green's function can be decomposed into its longitudinal and transverse components $G\left(\mathbf{r}-\mathbf{r}^{\prime}, \omega\right)=G_{\|}\left(\mathbf{r}-\mathbf{r}^{\prime}\right)+$ $G_{\perp}\left(\mathbf{r}-\mathbf{r}^{\prime}, \omega\right)$ which separates the static and dynamic aspects of the system. The longitudinal component reads $[35,60]$

$$
G_{\|}\left(\mathbf{r}-\mathbf{r}^{\prime}\right)=-\frac{4 a^{3}}{L} \sum_{m=1}^{\infty} k_{m}^{2} K_{0}\left(k_{m}\left|\mathbf{r}-\mathbf{r}^{\prime}\right|\right),
$$

and describes the instantaneous Coulomb field generated by a point dipole source, where $K_{0}$ is the modified Bessel function of zeroth order and second kind which decays like $K_{0}(x) \sim$ $e^{-x} / \sqrt{x}$. Therefore, the longitudinal Green's function encodes the short-range Coulomb interactions between the dipoles whose strength decreases rapidly with the separation distance as shown in Fig. 1(e).

Furthermore, the dipoles also couple to the transverse photonic modes of the cavity waveguide which can mediate long-range interactions between the dipoles. Since we are interested in the regime of cavity widths $L<\lambda_{0}$, we retain only the dominant contribution from the fundamental transverse electromagnetic (TEM) cavity mode. The corresponding transverse Green's function reads $[35,60]$

$$
G_{\perp}^{\mathrm{TEM}}\left(\mathbf{r}-\mathbf{r}^{\prime}, \omega\right)=i \frac{\pi a^{3} k_{\omega}^{2}}{L} H_{0}^{(1)}\left(k_{\omega}\left|\mathbf{r}-\mathbf{r}^{\prime}\right|\right),
$$

and the Hankel function decays like $H_{0}^{(1)}(x) \sim e^{i x} / \sqrt{x}$. Therefore, the real part of the transverse Green's function describes coherent long-range interactions mediated by the cavity photons whose strength oscillates and decreases slowly with the separation distance as shown in Fig. 1(f).

For large cavity widths $\left(L \sim \lambda_{0}\right)$, the essential physics near the Dirac points is dominated by the Coulomb interactions due to the subwavelength spacing of metasurface. However, for small cavity widths $\left(L \ll \lambda_{0}\right)$, the Coulomb interactions are exponentially suppressed due to the screening effect of the cavity waveguide. In stark contrast, the strength of the photon-mediated interactions increases as the cavity width is reduced. This is because the gapless TEM mode has no cut-off frequency and the reducing mode volume increases the lightmatter interaction strength. Consequently, for small cavity widths the essential physics is dominated by the photonmediated interactions. In what follows we show that this transition of the dominant dipolar coupling has a dramatic 
effect on the geometrical and topological properties of the polaritons.

\section{CAVITY POLARITONS}

We can exploit the periodicity of the metasurface by introducing the Fourier transform of the dipole moments

$$
\tilde{p}_{i}(\mathbf{q}, \omega)=\frac{\sqrt{\mathcal{A}}}{2 \pi} \sum_{\mathbf{R}} p_{\mathbf{R}_{i}}(\omega) e^{-i \mathbf{q} \cdot \mathbf{R}_{i}},
$$

where $\mathbf{q}=\left[q_{x}, q_{y}\right]$ is the Bloch wave vector that is restricted to the first Brillouin zone, and $\mathcal{A}=2 \sqrt{3} a^{2}$ is the area of the unit cell. We can then recast the coupled-dipole equations into $3 \times 3$ matrix eigenvalue equations in momentum space

$$
\frac{1}{\check{\alpha}(\omega)}|\varphi(\mathbf{q})\rangle=\left[\mathcal{D}_{\|}(\mathbf{q})+\mathcal{D}_{\perp}(\mathbf{q}, \omega)\right]|\varphi(\mathbf{q})\rangle,
$$

where $\quad \check{\alpha}^{-1}(\omega)=\alpha_{\mathrm{B}}^{-1}(\omega)-\operatorname{Re}[\Sigma(\omega)] \quad$ and $\quad|\varphi(\mathbf{q})\rangle=$ $\left[\widetilde{p}_{\mathrm{A}}(\mathbf{q}), \widetilde{p}_{\mathrm{B}}(\mathbf{q}), \widetilde{p}_{\mathrm{C}}(\mathbf{q})\right]$ is the vector of Fourier variables.

Furthermore, $\mathcal{D}_{\|}(\mathbf{q})$ is the longitudinal dynamical matrix which encodes the frequency shifts due to the Coulomb interactions, and the matrix elements are given by

$$
\mathcal{D}_{\|}^{i j}(\mathbf{q})=\sum_{\mathbf{R}} G_{\|}\left(\mathbf{R}+\mathbf{d}_{i}-\mathbf{d}_{j}\right) e^{-i \mathbf{q} \cdot\left(\mathbf{R}+\mathbf{d}_{i}-\mathbf{d}_{j}\right)}-\delta_{i j} G_{\|}(0) .
$$

Since the Coulomb interactions are short range, these lattice sums converge rapidly in real space. Note that the second term in Eq. (14) removes the divergent term in the sum $(\mathbf{R}=0)$ for the intrasublattice matrix elements.

Moreover, $\mathcal{D}_{\perp}(\mathbf{q}, \omega)$ is the transverse dynamical matrix which encodes the frequency shifts due to the photonmediated interactions, and the matrix elements are given by

$$
\mathcal{D}_{\perp}^{i j}(\mathbf{q}, \omega)=\sum_{\mathbf{g}} \frac{\omega^{2} \xi^{2} \phi_{\mathbf{q}}^{i j}}{\omega_{\mathbf{q}-\mathbf{g}}^{2}-\omega^{2}}-\delta_{i j} \operatorname{Re}\left[G_{\perp}^{\mathrm{TEM}}(0, \omega)\right] .
$$

Since the photon-mediated interactions are long range, here we have used the Poisson summation technique to convert the sum over lattice vectors to a rapidly converging sum over reciprocal lattice vectors $[35,60]$. Furthermore, $\xi=\sqrt{4 \pi a^{3} / \mathcal{A L}}$ parametrizes the strength of the light-matter coupling to the TEM cavity mode which has a linear dispersion $\omega_{\mathbf{q}-\mathbf{g}}=c \mid \mathbf{q}-$ $\mathbf{g} \mid$, and the phase factors

$$
\phi_{\mathbf{g}}^{i j}=e^{i \mathbf{g} \cdot\left(\mathbf{d}_{j}-\mathbf{d}_{i}\right)}
$$

arise due the non-Bravais nature of the lattice. Note that although the intrasublattice matrix elements in Eq. (15) are well defined, the two terms separately diverge. Therefore one needs to use the regularization procedure outlined in Appendix A to numerically evaluate the matrix elements.

\section{A. Cavity polariton dispersion}

We can simplify Eq. (13) by linearizing the eigenvalue problem which captures the essential physics away from the light-line. To do this we evaluate the polarizability correction and the transverse dynamical matrix at the renormalized cavity resonant frequency $\omega_{\text {cav }}$ which is given by
$\operatorname{Re}\left[\check{\alpha}^{-1}\left(\omega_{\text {cav }}\right)\right]=0$. We can then obtain the polariton dispersion by solving the following characteristic equation

$$
\operatorname{det}\left[\left(\omega^{2}-\omega_{\text {cav }}^{2}\right) \mathbb{1}_{3}+2 \omega_{0} \mu \mathcal{D}_{\|}(\mathbf{q})+2 \omega_{0} \mu \mathcal{D}_{\perp}\left(\mathbf{q}, \omega_{\text {cav }}\right)\right]=0,
$$

where $\mathbb{1}_{3}$ is the $3 \times 3$ identity matrix and we have neglected nonradiative losses for simplicity.

In Fig. 2(a) we show the polariton dispersion for the shrunken metasurface $(\delta=0.05)$ with a large cavity width where the Coulomb interactions are dominant (solid line). Note that in this panel we have neglected the weak photonmediated interactions which have a negligible effect near the $\mathrm{K} / \mathrm{K}^{\prime}$ valleys (see Appendix $\mathrm{C}$ for more details). In Fig. 2(b) we show the polariton dispersion for the same shrunken metasurface but now with a small cavity width where the photon-mediated interactions are dominant (solid line). In both interaction regimes, the symmetry-reducing perturbation removes the deterministic Dirac points exhibited by the unperturbed kagome metasurface (dashed lines), thereby opening a gap at the $\mathrm{K} / \mathrm{K}^{\prime}$ points. While they share this feature, the spectra in the two regimes look qualitatively different, for example, the (approximate) flat band is above/below the Dirac point for the small/large cavity width.

\section{B. Eigenstates at the $K / K^{\prime}$ points}

To further elucidate the difference between the two regimes, we can study the eigenstates at the $\mathrm{K}(\tau=+)$ and $\mathrm{K}^{\prime}$ ( $\tau=-$ ) points located at $\tau \mathbf{K}=\tau[2 \pi / 3 a, 0]$, where $\tau= \pm$ is the valley index. Because the symmetry-reducing perturbation preserves the $C_{3}$ symmetry of the metasurface, the eigenstates at the $\mathrm{K} / \mathrm{K}^{\prime}$ points will be simultaneous eigenstates of the $C_{3}$ operator

$$
\mathcal{U}_{C_{3}}^{\tau}\left|\varphi_{\tau}^{l}\right\rangle=e^{i \frac{2 \pi}{3} l}\left|\varphi_{\tau}^{l}\right\rangle .
$$

Here the unitary operator

$$
\mathcal{U}_{C_{3}}^{\tau}=\left[\begin{array}{ccc}
0 & 0 & e^{-i \frac{\pi}{3}(1-\delta) \tau} \\
e^{-i \frac{\pi}{3}(1-\delta) \tau} & 0 & 0 \\
0 & e^{i \frac{2 \pi}{3}(1-\delta) \tau} & 0
\end{array}\right]
$$

represents a rotation of $2 \pi / 3$ about the center of the unit cell, $\left|\varphi_{\tau}^{l}\right\rangle$ are the corresponding eigenstates, and $l=0, \pm 1$ are the pseudoangular momentum eigenvalues. The eigenstates at the $\mathrm{K}$ point therefore read

$$
\begin{aligned}
& \left|\varphi_{+}^{0}\right\rangle=\frac{1}{\sqrt{3}}\left[1, e^{-i \frac{\pi}{3}(1-\delta)}, e^{i \frac{\pi}{3}(1-\delta)}\right]^{\mathrm{T}}, \\
& \left|\varphi_{+}^{-}\right\rangle=\frac{1}{\sqrt{3}}\left[1, e^{i \frac{\pi}{3}(1+\delta)}, e^{-i \frac{\pi}{3}(1+\delta)}\right]^{\mathrm{T}}, \\
& \left|\varphi_{+}^{+}\right\rangle=\frac{1}{\sqrt{3}}\left[-1, e^{i \frac{\pi}{3} \delta}, e^{-i \frac{\pi}{3} \delta}\right]^{\mathrm{T}},
\end{aligned}
$$

and in Fig. 2(c) we schematically depict the corresponding dipole distributions within a unit cell, where $+/-$ corresponds to anticlockwise/clockwise phase vortices. Furthermore, the eigenstates at the $\mathrm{K}^{\prime}$ point are related via time-reversal symmetry and are given by $\left|\varphi_{-}^{0}\right\rangle=\left|\varphi_{+}^{0}\right\rangle^{*},\left|\varphi_{-}^{-}\right\rangle=\left|\varphi_{+}^{+}\right\rangle^{*}$, and $\left|\varphi_{-}^{+}\right\rangle=\left|\varphi_{+}^{-}\right\rangle^{*}$. 
(a)

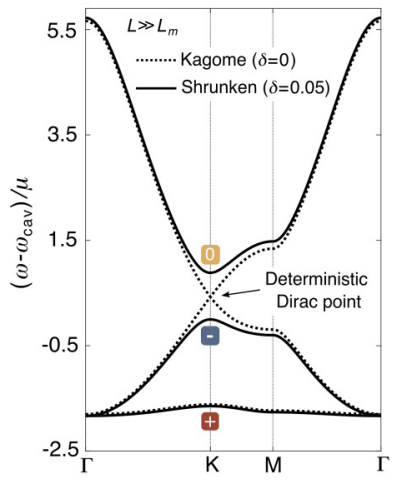

(b)

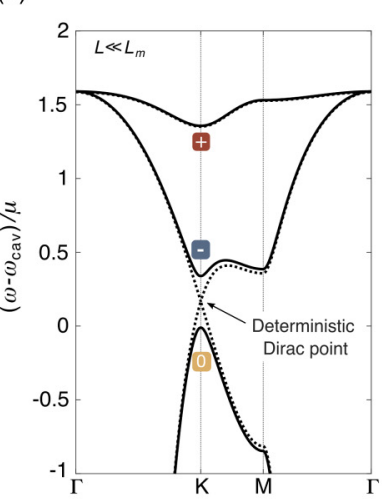

(c)
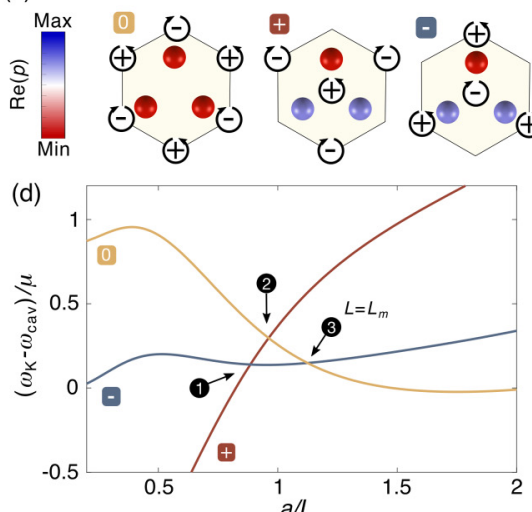

(e)

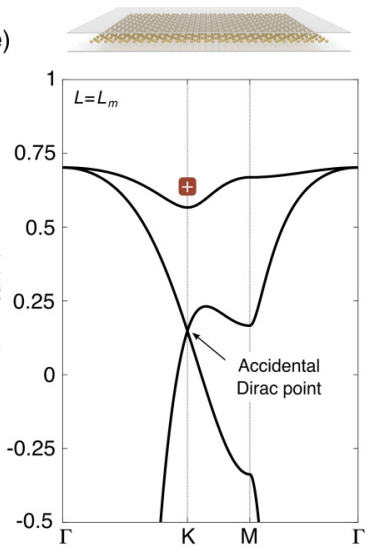

FIG. 2. (a), (b) Polariton dispersion for the kagome metasurface (dashed lines) and shrunken metasurface (solid lines) with $L=5 a$ and $L=0.5 a$, respectively. The symmetry-reducing perturbation removes the deterministic Dirac points, opening a gap at the $\mathrm{K} / \mathrm{K}^{\prime}$ points. The bands at the $\mathrm{K}$ point are labeled according to the pseudoangular momentum number of the eigenstates whose corresponding dipole distributions are schematically depicted in panel (c). Here $+/-$ denote anticlockwise/clockwise phase vortices which are located at the $C_{3}$ rotation centers. (d) Frequency evolution of the eigenstates at the $\mathrm{K}$ point as the cavity width is varied for the shrunken metasurface, where we observe three band inversions (labeled 1-3). (e) Polariton dispersion at the critical cavity width $\left(L=L_{m}\right)$ which exhibits accidental Dirac points. Results obtained with $\delta=0.05, a=0.1 \lambda_{0}$, and $\mu=0.001 \omega_{0}$.

\section{Cavity-induced band inversions}

In Fig. 2(a) and Fig. 2(b) we label the polariton bands at the $\mathrm{K}$ point according to the pseudoangular momentum number of the eigenstates. Interestingly, for the same symmetry-breaking parameter $(\delta=0.05)$, the ordering of the eigenstates is entirely reversed for the two regimes. To elucidate this further, in Fig. 2(d) we show the frequency evolution of the eigenstates at the $\mathrm{K}$ point as the cavity width is reduced. Due to the competition between the Coulomb and photon-mediated interactions, we observe three band inversions. The first two band inversions result in the $l=+1$ eigenstate evolving from being the lowest in frequency to the highest. However, here we are particularly interested in the third band inversion. At this critical cavity width $L=L_{m}$, the degeneracy between the $l=$ 0 and the $l=-1$ eigenstates is restored, despite the reduced symmetry of the metasurface. Consequently, the polariton dispersion exhibits accidental Dirac points at this critical cavity width as shown in Fig. 2(e).

\section{CAVITY-INDUCED TOPOLOGICAL TRANSITIONS}

To characterize the essential topology related to the third band inversion, we will derive an effective two-band Hamiltonian that describes the polaritons near the $\mathrm{K} / \mathrm{K}^{\prime}$ valleys within the subspace spanned by the degenerate eigenstates of the unperturbed lattice (see Appendix B for the derivation and analytic expressions for the parameters). The effective Hamiltonian reads $(\hbar=1)$

$$
\mathcal{H}_{\tau}=\omega_{\mathrm{D}}(L) \mathbb{1}_{2}+v_{\mathrm{D}}(L)\left(\tau \sigma_{x} k_{x}+\sigma_{y} k_{y}\right)+m(L) \sigma_{z},
$$

where $\mathbf{k}=\left[k_{x}, k_{y}\right]$ is the wave vector measured from the $\mathrm{K} / \mathrm{K}^{\prime}$ point. Furthermore, $\mathbb{1}_{2}$ is the $2 \times 2$ identity matrix and $\sigma_{i}$ are the Pauli matrices that act in the $\left\{\left|\varphi_{+}^{0}\right\rangle,\left|\varphi_{+}^{-}\right\rangle\right\}$and $\left\{\left|\varphi_{-}^{0}\right\rangle,\left|\varphi_{-}^{+}\right\rangle\right\}$subspace for the $\mathrm{K}$ and $\mathrm{K}^{\prime}$ valleys, respectively. Equation (21) is equivalent to a massive Dirac Hamiltonian where the corresponding polariton spectrum is

$$
\omega_{\lambda}(\mathbf{k})=\omega_{\mathrm{D}}+\lambda \sqrt{v_{\mathrm{D}}^{2} k^{2}+m^{2}},
$$

as schematically depicted in Fig. 3(a). Here $\lambda= \pm$ labels the upper $(\lambda=+)$ and lower $(\lambda=-)$ effective polariton bands, $\omega_{\mathrm{D}}$ is the Dirac frequency, $v_{\mathrm{D}}$ is the Dirac velocity, and $m$ is the Dirac mass which determines the size of the gap. Note that the effective Hamiltonian is only valid when the third band is separated in frequency and, therefore, it captures the essential physics before and after the first two band inversions.

\section{A. Inverting the sign of the Dirac mass}

We can split the Dirac mass into two distinct contributions $m=m_{\|}+m_{\perp}$, where the longitudinal mass reads

$$
\begin{aligned}
m_{\|}= & \frac{\mu}{\sqrt{3}} \frac{\omega_{0}}{\omega_{\mathrm{cav}}} \sum_{\mathbf{R}} \operatorname{Re}\left[i G_{\|}\left(\mathbf{R}+\mathbf{d}_{\mathrm{A}}-\mathbf{d}_{\mathrm{B}}\right) e^{i \frac{\pi}{3} \delta} e^{-i \mathbf{q} \cdot\left(\mathbf{R}+\mathbf{d}_{\mathrm{A}}-\mathbf{d}_{\mathrm{B}}\right)}\right. \\
& -i G_{\|}\left(\mathbf{R}+\mathbf{d}_{\mathrm{A}}-\mathbf{d}_{\mathrm{C}}\right) e^{-i \frac{\pi}{3} \delta} e^{-i \mathbf{q} \cdot\left(\mathbf{R}+\mathbf{d}_{\mathrm{A}}-\mathbf{d}_{\mathrm{C}}\right)} \\
& \left.-i G_{\|}\left(\mathbf{R}+\mathbf{d}_{\mathrm{B}}-\mathbf{d}_{\mathrm{C}}\right) e^{-i \frac{2 \pi}{3} \delta} e^{-i \mathbf{q} \cdot\left(\mathbf{R}+\mathbf{d}_{\mathrm{B}}-\mathbf{d}_{\mathrm{C}}\right)}\right]
\end{aligned}
$$

and the transverse mass reads

$$
\begin{aligned}
m_{\perp}= & \frac{\mu}{\sqrt{3}} \frac{\omega_{0}}{\omega_{\mathrm{cav}}} \sum_{\mathbf{g}} \frac{\omega_{\mathrm{cav}}^{2} \xi^{2}}{\omega_{\mathbf{K}-\mathbf{g}}^{2}-\omega_{\mathrm{cav}}^{2}} \operatorname{Re}\left[i e^{i \frac{\pi}{3} \delta} \phi_{\mathbf{g}}^{\mathrm{AB}}\right. \\
& \left.-i e^{-i \frac{\pi}{3} \delta} \phi_{\mathbf{g}}^{\mathrm{AC}}-i e^{-i \frac{2 \pi}{3} \delta} \phi_{\mathbf{g}}^{\mathrm{BC}}\right] .
\end{aligned}
$$

These encode the gap that is generated by the Coulomb and photon-mediated interactions, respectively. For the unperturbed kagome metasurface $(\delta=0)$, the symmetry forces both the longitudinal and the transverse mass to vanish separately $m_{\|}=m_{\perp}=0$, which results in deterministic Dirac points for all cavity widths.

In Fig. 3(b) we show how these masses evolve as the cavity width is varied for a fixed shrunken metasurface $(\delta=0.05)$. Crucially, the longitudinal (dashed orange line) and transverse 
(a)

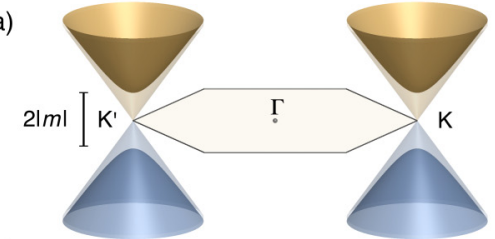

(b)

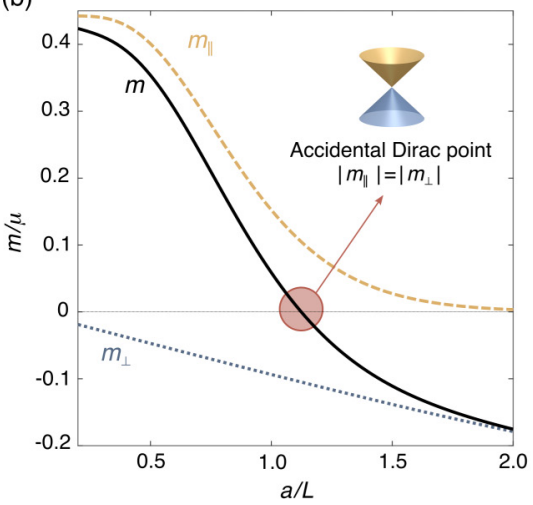

(c)

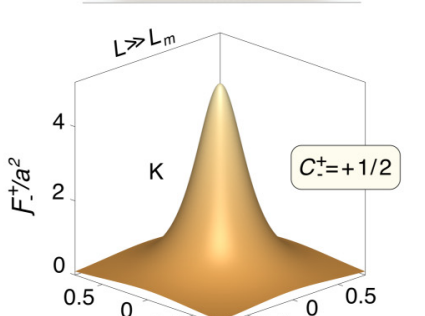

(d)
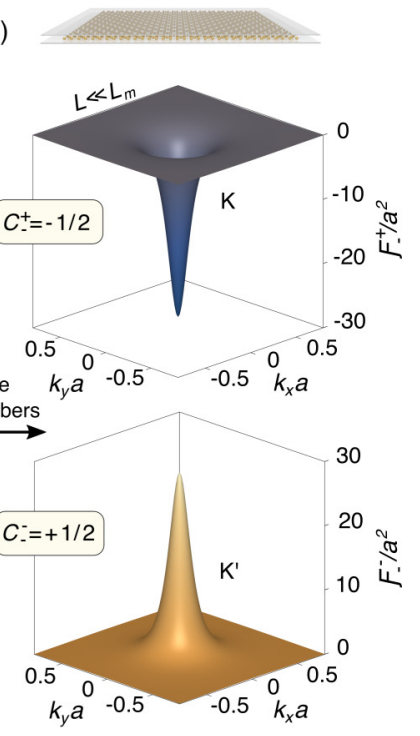

FIG. 3. (a) Schematic of the massive Dirac cone spectrum that is generated by the symmetry-reducing perturbation, where the size of the gap is determined by the Dirac mass. (b) Evolution of the longitudinal (dashed orange line), transverse (dotted blue line) and total (solid black line) Dirac mass as the cavity width is varied for the shrunken metasurface. While the longitudinal and transverse masses never vanish, they have opposite signs and thus tend to compensate each other. At the critical width $\left(L=L_{m}\right)$ they perfectly cancel and the total mass vanishes, leading to accidental Dirac points where the Dirac mass changes sign. (c) Localized Berry curvature corresponding to the lower band $(\lambda=-)$ near the $\mathrm{K}$ (top) and $\mathrm{K}^{\prime}$ (bottom) valleys for the shrunken metasurface with $L=5 a$. We also show the corresponding valley-Chern numbers. (d) Same as panel (c) but for $L=0.5 a$. The transition of the dominant dipolar coupling from Coulomb to photon-mediated interactions switches the sign of the Berry curvature within each valley and thus inverts the valley-Chern numbers. Results obtained with $\delta=0.05, a=0.1 \lambda_{0}$ and $\mu=0.001 \omega_{0}$.

(dotted blue line) masses do not vanish for any cavity width due to the reduced symmetry; however, they have opposite signs and thus tend to compensate each other. Moreover, the longitudinal mass dominates for large cavity widths, while the transverse mass dominates for small cavity widths. At the critical cavity width $L=L_{\mathrm{m}}$ they perfectly cancel $\left(\left|m_{\|}\right|=\left|m_{\perp}\right|\right)$ and the Dirac mass vanishes (solid black line), giving rise to accidental Dirac points. Therefore, by varying only the cavity width, one can induce a topological band inversion where the band gap closes and reopens, switching the sign of the Dirac mass.

\section{B. Cavity-induced inversion of the valley-Chern numbers}

Using the effective Dirac Hamiltonian, the Berry curvature for the $\lambda$ band reads

$$
\mathcal{F}_{\lambda}^{\tau}(\mathbf{k})=-\tau \lambda \frac{m v_{\mathrm{D}}^{2}}{2\left(v_{\mathrm{D}}^{2}|\mathbf{k}|^{2}+m^{2}\right)^{3 / 2}},
$$

and the corresponding valley-Chern numbers are given by

$$
C_{\lambda}^{\tau}=\frac{1}{2 \pi} \iint_{\mathbb{R}^{2}} d^{2} \mathbf{k} \mathcal{F}_{\lambda}^{\tau}(\mathbf{k})=-\tau \lambda \operatorname{sgn}(m) \frac{1}{2} .
$$

This approximation is valid when the third band is well separated in frequency and for small perturbations such that the Berry curvature is localized near the $\mathrm{K} / \mathrm{K}^{\prime}$ points (see Appendix D for more details).
In Fig. 3(c) we show the Berry curvature and the valleyChern numbers corresponding to the lower band $(\lambda=-)$ for the shrunken metasurface $(\delta=0.05)$ with a large cavity width. In Fig. 3(d) we show the Berry curvature and valleyChern numbers for the same shrunken metasurface but now with a small cavity width. Remarkably, the Berry curvature changes sign due to the change in sign of the Dirac mass and, as a result, the valley-Chern numbers become inverted in the two regimes.

\section{SWITCHING THE CHIRALITY OF THE POLARITON VALLEY-HALL EDGE STATES}

Although the valley-Chern numbers are not strictly topological invariants, there exists a bulk-boundary correspondence for a domain-wall interface between two regions with opposite valley-Chern numbers [26-33]. Provided that the interface does not mix the two valleys and the Berry curvature remains localized near the $\mathrm{K} / \mathrm{K}^{\prime}$ points, then the change in valley-Chern number across the interface $\left(\Delta C_{-}^{\tau}\right)$ determines the number of valley-polarized chiral edge states.

To see how the cavity modifies the polariton valley-Hall edge states, we consider the interface shown in Fig. 4(a) where region $\mathrm{A}$ is a shrunken metasurface $\left(\delta_{\mathrm{A}}=0.05\right)$ and region $\mathrm{B}$ is an expanded metasurface $\left(\delta_{\mathrm{B}}=-0.05\right)$. While the bulk polariton dispersion is identical in both regions, they have opposite Dirac masses $\left(m_{\mathrm{B}}=-m_{\mathrm{A}}\right)$ and therefore opposite valley-Chern numbers. 
(a)

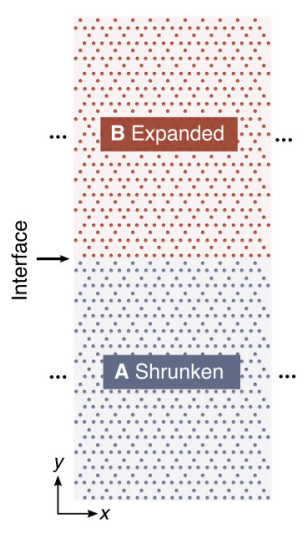

(b)

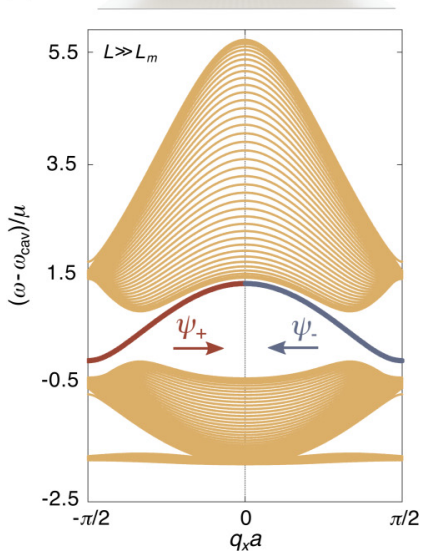

(c)

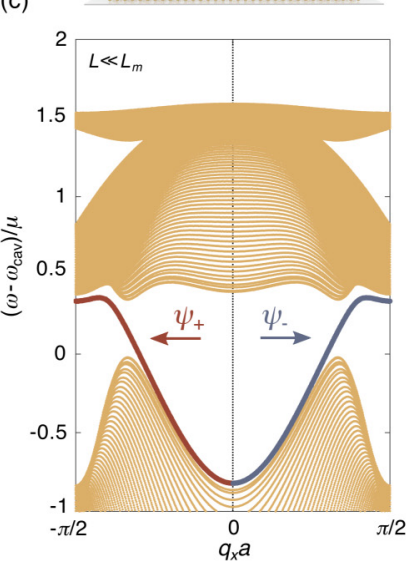

(d)

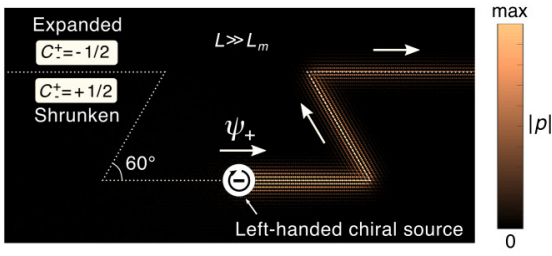

(e)

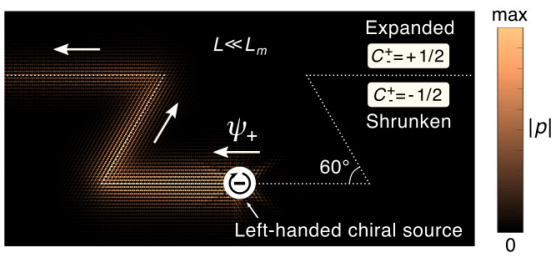

FIG. 4. (a) Schematic of a domain-wall interface between two regions with opposite valley-Chern numbers, where region $\mathrm{A}$ is a shrunken metasurface $\left(\delta_{\mathrm{A}}=0.05\right)$ and region $\mathrm{B}$ is an expanded metasurface $\left(\delta_{\mathrm{B}}=-0.05\right)$. (b) Interface dispersion for $L=5 a$. The orange bands correspond to the projected bulk states, while the red/blue bands correspond to the valley-Hall edge states $\psi_{+} / \psi_{-}$in the $\mathrm{K} / \mathrm{K}^{\prime}$ valleys which propagate in opposite directions by virtue of time-reversal symmetry. (c) Same as panel (b) but for $L=0.5 a$. The propagation direction of the valley-Hall edge states is reversed due to the cavity-induced inversion of the valley-Chern numbers which switches their chirality. (d), (e) Steady-state distribution of the dipole moments for an $\Omega$-shaped interface (white dotted line) with $L=5 a$ and $L=0.5 a$, respectively. We excite the metasurface with a left-handed chiral source which is fixed near the interface such that it selectively couples to the $\mathrm{K}$ valley edge state in both regimes. One observes that the edge state is not backscattered by the sharp bends, and the propagation direction is reversed in the two regimes due to the cavity-induced inversion of the valley-Chern numbers. Results obtained with $a=0.1 \lambda_{0}$ and $\mu=0.001 \omega_{0}$. In panels (d) and (e) we set $\gamma_{\mathrm{nr}}=0.02 \mu$.

\section{A. Polariton valley-Hall edge states}

Before we numerically calculate the interface dispersion using the coupled-dipole equations, we can gain some analytical insight using the effective Dirac Hamiltonian in real space,

$$
\mathcal{H}_{\tau}=\omega_{\mathrm{D}} \mathbb{1}_{2}-i v_{\mathrm{D}}\left(\tau \sigma_{x} \partial_{x}+\sigma_{y} \partial_{y}\right)+m(y) \sigma_{z},
$$

where the interface is modeled by a spatially varying mass,

$$
m(y)=\left\{\begin{array}{ll}
+m_{\mathrm{A}}, & \text { for } y<0 \\
-m_{\mathrm{A}}, & \text { for } y>0
\end{array} .\right.
$$

Seeking the bounded solutions to Eq. (27), one finds that each valley supports one chiral edge state of the form

$$
\psi_{\tau}(\mathbf{r}) \propto\left[\begin{array}{c}
1 \\
\operatorname{sgn}\left(v_{\mathrm{D}}\right) \operatorname{sgn}\left(m_{\mathrm{A}}\right)
\end{array}\right] e^{i k_{x} x} e^{-\frac{\left|m_{\mathrm{A}}\right|}{\left|v_{\mathrm{D}}\right|}|y|},
$$

which are exponentially localized at the interface-this is expected from the bulk-boundary correspondence since the change in valley-Chern number is $\left|\Delta C_{-}^{\tau}\right|=1$. These edge states exhibit a linear dispersion

$$
\omega_{\tau}\left(k_{x}\right)=\omega_{\mathrm{D}}+\operatorname{sgn}\left(\Delta C_{-}^{\tau}\right)\left|v_{\mathrm{D}}\right| k_{x},
$$

where their group velocity is given by

$$
v_{\tau}=\frac{\partial \omega_{\tau}}{\partial k_{x}}=\operatorname{sgn}\left(\Delta C_{-}^{\tau}\right)\left|v_{\mathrm{D}}\right| .
$$

Therefore the sign of $\Delta C_{-}^{\tau}$ determines the chirality of the valley-Hall edge states. Since we have shown that one can invert the sign of the valley-Chern numbers by varying the cavity width, one can anticipate that this inversion will switch the propagation direction of the edge states along the interface.

\section{B. Interface dispersion}

To verify these analytical predictions we go beyond the approximations of the effective Dirac Hamiltonian and calculate the full dispersion for the interface using the coupled-dipole equations. We consider a supercell which contains 40 unit cells in each region and we apply periodic boundary conditions along both directions. However, since we focus on the A/B interface, for clarity we will remove the edge states associated with the $\mathrm{B} / \mathrm{A}$ interface.

In Fig. 4(b) we show the interface dispersion for a large cavity width. Note that for clarity we have again neglected the weak photon-mediated interactions which have a negligible effect near the $\mathrm{K} / \mathrm{K}^{\prime}$ valleys (see Appendix $\mathrm{C}$ for more details). The orange bands correspond to the projected bulk states, while the red/blue bands correspond to the spectrum of the valley-Hall edge states near the $\mathrm{K} / \mathrm{K}^{\prime}$ valleys.

Since $\Delta C_{-}^{\tau}=\tau$ for large cavity widths, the $\mathrm{K}$ valley edge states $\left(\psi_{+}\right)$propagate to the right (red band) while the the $\mathrm{K}^{\prime}$ valley edge states $\left(\psi_{-}\right)$propagate to the left (blue band). In Fig. 4(c) we show the dispersion for the same interface but now with a small cavity width. Due to the cavity-induced inversion of the valley-Chern numbers, we now have $\Delta C_{-}^{\tau}=$ $-\tau$ and, therefore, the propagation direction of the polariton valley-Hall edge states is reversed.

\section{Selective excitation of the valley-Hall edge states}

Finally, we show that one can selectively excite the valleyHall edge states with a chiral source and route them around sharp corners. Specifically, we consider an $\Omega$-shaped interface between a shrunken and expanded metasurface which contains multiple sharp bends. Due to the lack of translational 
invariance, we have to numerically solve a large system of coupled dipole equations in real space.

We excite the metasurface with a left-handed chiral source which is comprised of six point dipole sources with a fixed phase difference. The corresponding driving field reads

$$
E_{0}(\mathbf{r}) \propto \sum_{m=1}^{6} e^{-i \frac{m \pi}{3}} G\left(\mathbf{r}-\mathbf{r}_{0}-\mathbf{e}_{m}, \omega\right),
$$

where $\mathbf{e}_{m}=0.01 a[\cos (m \pi / 3), \sin (m \pi / 3)]$. We chose the driving frequencies such that they correspond to the middle of the bulk gap at the $\mathrm{K} / \mathrm{K}^{\prime}$ points. Furthermore, it is important to stress that the Dirac velocity also has opposite signs in the two limiting regimes, so the spinors in Eq. (29) within each valley remain the same for the large and small cavity widths. Therefore, we can fix the position of the chiral source $\left(\mathbf{r}_{0}\right)$ close to the interface so that it selectively excites only the topological edge states in the $\mathrm{K}$ valley in both regimes. Finally, we set the nonradiative losses to $\gamma_{\mathrm{nr}}=0.02 \mu$.

In Fig. 4(d) we plot the steady-state amplitude of the dipole moments for a large cavity width where the Coulomb interactions are dominant. One clearly observes the chiral nature of the valley-Hall edge state which only propagates to the right and can be routed around the sharp corners without any significant backscattering. In Fig. 4(e) we show the simulation for the same interface but now with a small cavity width where the photon-mediated interactions are dominant. As predicted, the valley-Hall edge state now propagates in the opposite direction due to the cavity-induced inversion of the valley-Chern numbers thus verifying the ability to switch their chirality by varying only the cavity width.

\section{OUTLOOK}

In this work we have shown that the geometrical and topological properties of the polaritons depend sensitively on the local photonic environment due to their mixed light-matter character. While the symmetry-reducing perturbation is required to induce the valley-Hall phase, we have demonstrated that one can engineer topological transitions by structuring the photonic environment. In particular, we have shown that one can tune the Dirac mass and invert the valley-Chern numbers by embedding the metasurface inside a cavity waveguide which modifies the nature of the dipole-dipole interactions.

While we have considered a minimal theoretical model one could generalize the theory to include more complex antennas that exhibit magnetic dipole or higher-order multipole moments, such as split-ring resonators or dielectric Mie resonators. Furthermore, while we have focused on a cavity waveguide there are other ways to structure the photonic environment which may be more suitable for certain experimental realizations and could have other advantages. For example, one could interface the emitters/antennas with a graphene membrane [61] where the interactions will be mediated by surface plasmons whose properties can be tuned via a gate voltage. Moreover, one could interface them with a photonic crystal where the interactions will be mediated by the guided modes $[43,62]$. Here one has the freedom to engineer the photonic band structure which could be tuned via electrooptical or nonlinear effects. These may provide alternative ways to induce topological transitions in arrays of interacting emitters/antennas.

Finally, the underlying principle presented in this work could also have implications for other topological phases that have recently attracted considerable interest in photonics. For example, perturbed kagome lattices have also been shown to exhibit a higher-order topological phase with topological corner states where it is usually asserted that the shrunken/expanded lattice is the trivial/topological phase [59]. However, here we have shown the ability to induce multiple band inversions without ever modifying the lattice geometry of the metasurface; do these band inversions change the topological phase? If so, what is the fate of the topological corner states as the photonic environment is modified?

\section{ACKNOWLEDGMENTS}

C.-R.M acknowledges valuable discussions with S.A.R. Horsley and financial support from the Rank Prize Funds and the Engineering and Physical Sciences Research Council (EPSRC) of the United Kingdom through the EPSRC Centre for Doctoral Training in Metamaterials (Grant No. EP/L015331/1). E.M. acknowledges financial support from the Royal Society International Exchanges Grant IEC/R2/192166.

\section{APPENDIX A: REGULARIZATION OF THE DIVERGENT TERMS}

Here we outline the regularization procedure that enables one to evaluate the transverse dynamical matrix elements in Eq. (15). We regularize the large momentum behavior of the TEM Green's function by introducing a Gaussian cut-off function into its integral representation $[35,60]$,

$\breve{G}_{\perp}^{\mathrm{TEM}}\left(\mathbf{r}-\mathbf{r}^{\prime}, \omega\right)=\frac{4 \pi a^{3} k_{\omega}^{2}}{L} \iint \frac{d^{2} \mathbf{k}}{(2 \pi)^{2}} \frac{e^{i \mathbf{k} \cdot\left(\mathbf{r}-\mathbf{r}^{\prime}\right)}}{k^{2}-k_{\omega}^{2}-i 0^{+}} e^{-\eta^{2} k^{2}}$,

where the infinitesimal imaginary term in the denominator ensures we have outgoing waves from the point source. We choose the cut-off parameter $\eta \ll a$ such that the regularization has a negligible effect on the interactions between dipoles. However, the Gaussian cut-off effectively smears out the divergent part of the transverse Green's function over a small area, rendering it finite and well defined at the source location $[35,60]$

$$
\breve{G}_{\perp}^{\mathrm{TEM}}(0, \omega)=\frac{a^{3} k_{\omega}^{2}}{L} e^{-\eta^{2} k_{\omega}^{2}}\left[i \pi-\operatorname{Ei}\left(\eta^{2} k_{\omega}^{2}\right)\right],
$$

where $\operatorname{Ei}(x)=-P . V \cdot \int_{-x}^{\infty} \frac{e^{-w}}{w} \mathrm{~d} w$ is the exponential integral and P.V. denotes the Cauchy principal value. After inserting the Gaussian cut-off function, the regularized transverse dynamical matrix elements read

$$
\mathcal{D}_{\perp}^{i j}(\mathbf{q}, \omega)=\sum_{\mathbf{g}} \frac{\omega^{2} \xi^{2} \phi_{\mathbf{q}}^{i j}}{\omega_{\mathbf{q}-\mathbf{g}}^{2}-\omega^{2}} e^{-\eta^{2}|\mathbf{q}-\mathbf{g}|^{2}}-\delta_{i j} \operatorname{Re}\left[\breve{G}_{\perp}^{\mathrm{TEM}}(0, \omega)\right] .
$$




\section{APPENDIX B: EFFECTIVE TWO-BAND HAMILTONIAN NEAR THE K/K' POINTS}

Here we outline the derivation of the effective two-band Hamiltonian in Eq. (21), and we focus on the K point because the equivalent Hamiltonian near the $\mathrm{K}^{\prime}$ point is related via time-reversal symmetry. To leading order in $\mathbf{k}=\mathbf{q}-\mathbf{K}$, the elements of the longitudinal dynamical matrix in Eq. (14) expand as

$$
\begin{aligned}
\mathcal{D}_{\|,+}^{i j}(\mathbf{k})= & \sum_{\mathbf{R}} G_{\|}\left(\mathbf{R}+\mathbf{d}_{i}-\mathbf{d}_{j}\right) e^{-i \mathbf{K} \cdot\left(\mathbf{R}+\mathbf{d}_{i}-\mathbf{d}_{j}\right)} \\
& -\delta_{i j} G_{\|}(0)-i k_{\nu} \sum_{\mathbf{R}}\left(\mathbf{R}+\mathbf{d}_{i}-\mathbf{d}_{j}\right)_{\nu} \\
& \times G_{\|}\left(\mathbf{R}+\mathbf{d}_{i}-\mathbf{d}_{j}\right) e^{-i \mathbf{K} \cdot\left(\mathbf{R}+\mathbf{d}_{i}-\mathbf{d}_{j}\right)},
\end{aligned}
$$

where $v=x, y$, while the elements of the transverse dynamical matrix in Eq. (15) expand as

$$
\begin{aligned}
\mathcal{D}_{\perp,+}^{i j}(\mathbf{k}, \omega)= & \sum_{\mathbf{g}} \frac{\omega^{2} \xi^{2} \phi_{\mathbf{g}}^{i j}}{\omega_{\mathbf{K}-\mathbf{g}}^{2}-\omega^{2}}-\delta_{i j} \operatorname{Re}\left[G_{\perp}^{\mathrm{TEM}}(0, \omega)\right] \\
& -k_{\nu} \sum_{\mathbf{g}} \frac{2 c^{2} \omega^{2} \xi^{2} \phi_{\mathbf{g}}^{i j}}{\left(\omega_{\mathbf{K}-\mathbf{g}}^{2}-\omega^{2}\right)^{2}}(\mathbf{K}-\mathbf{g})_{\nu} .
\end{aligned}
$$

Next we reduce the $3 \times 3$ matrix eigenvalue equation to an effective $2 \times 2$ matrix eigenvalue equation in the $\left\{\left|\varphi_{+}^{0}\right\rangle,\left|\varphi_{+}^{-}\right\rangle\right\}$ subspace:

$$
\frac{1}{\check{\alpha}(\omega)}\left|\psi_{+}(\mathbf{k})\right\rangle=\mathcal{D}_{+}^{\mathrm{eff}}(\mathbf{k}, \omega)\left|\psi_{+}(\mathbf{k})\right\rangle .
$$

Here the new basis is related to the sublattice basis via

$$
\left|\psi_{+}(\mathbf{k})\right\rangle=\left[\begin{array}{c}
\left\langle\varphi_{+}^{0} \mid \varphi(\mathbf{k})\right\rangle \\
\left\langle\varphi_{+}^{-} \mid \varphi(\mathbf{k})\right\rangle
\end{array}\right],
$$

and the effective dynamical matrix is given by

$$
\mathcal{D}_{+}^{\mathrm{eff}}(\mathbf{k}, \omega)=\left[\begin{array}{ll}
\left\langle\varphi_{+}^{0}\left|\mathcal{D}_{+}\right| \varphi_{+}^{0}\right\rangle & \left\langle\varphi_{+}^{0}\left|\mathcal{D}_{+}\right| \varphi_{+}^{-}\right\rangle \\
\left\langle\varphi_{+}^{-}\left|\mathcal{D}_{+}\right| \varphi_{+}^{0}\right\rangle & \left\langle\varphi_{+}^{-}\left|\mathcal{D}_{+}\right| \varphi_{+}^{-}\right\rangle
\end{array}\right],
$$

where $\mathcal{D}_{+}^{i j}(\mathbf{k}, \omega)=\mathcal{D}_{\|,+}^{i j}(\mathbf{k})+\mathcal{D}_{\perp,+}^{i j}(\mathbf{k}, \omega)$. Finally, we linearize the effective eigenvalue equation in Eq. (B3) by evaluating the polarizability correction and effective dynamical matrix at the cavity resonant frequency, and we approximate $\omega_{\text {cav }}^{2}-\omega^{2} \simeq 2 \omega_{\text {cav }}\left(\omega_{\text {cav }}-\omega\right)$. This allows us to write a simplified eigenvalue equation

$$
\omega\left|\psi_{+}(\mathbf{k})\right\rangle=\mathcal{H}_{+}(\mathbf{k})\left|\psi_{+}(\mathbf{k})\right\rangle,
$$

where the effective Hamiltonian is given by

$$
\mathcal{H}_{+}(\mathbf{k})=\omega_{\text {cav }} \mathbb{1}_{2}-\mu \frac{\omega_{0}}{\omega_{\text {cav }}} \mathcal{D}_{+}^{\text {eff }}\left(\mathbf{k}, \omega_{\text {cav }}\right) .
$$

After evaluating the matrix elements in Eq. (B5) and performing similar analysis for the $\mathrm{K}^{\prime}$ valley in the $\left\{\left|\varphi_{-}^{0}\right\rangle,\left|\varphi_{-}^{+}\right\rangle\right\}$ subspace, we obtain the effective Hamiltonian in Eq. (21). The Dirac frequency can be decomposed as $\omega_{\mathrm{D}}=\omega_{\text {cav }}+\omega_{\|}+$ $\omega_{\perp}$, where the contribution from the Coulomb interactions is

$$
\begin{aligned}
\omega_{\|}= & -\mu \frac{\omega_{0}}{\omega_{\mathrm{cav}}} \sum_{\mathbf{R} \neq 0} G_{\|}(\mathbf{R}) e^{-i \mathbf{K} \cdot \mathbf{R}}-\frac{\mu}{3} \frac{\omega_{0}}{\omega_{\mathrm{cav}}} \sum_{\mathbf{R}} \operatorname{Re}\left[G_{\|}\left(\mathbf{R}+\mathbf{d}_{\mathrm{A}}-\mathbf{d}_{\mathrm{B}}\right) e^{i \frac{\pi}{3} \delta} e^{-i \mathbf{K} \cdot\left(\mathbf{R}+\mathbf{d}_{\mathrm{A}}-\mathbf{d}_{\mathrm{B}}\right)}\right. \\
& \left.+G_{\|}\left(\mathbf{R}+\mathbf{d}_{\mathrm{A}}-\mathbf{d}_{\mathrm{C}}\right) e^{-i \frac{\pi}{3} \delta} e^{-i \mathbf{K} \cdot\left(\mathbf{R}+\mathbf{d}_{\mathrm{A}}-\mathbf{d}_{\mathrm{C}}\right)}-G_{\|}\left(\mathbf{R}+\mathbf{d}_{\mathrm{B}}-\mathbf{d}_{\mathrm{C}}\right) e^{-i \frac{2 \pi}{3} \delta} e^{-i \mathbf{K} \cdot\left(\mathbf{R}+\mathbf{d}_{\mathrm{B}}-\mathbf{d}_{\mathrm{C}}\right)}\right],
\end{aligned}
$$

and the contribution from the photon-mediated interactions reads

$$
\begin{aligned}
\omega_{\perp}= & \mu \frac{\omega_{0}}{\omega_{\mathrm{cav}}} \operatorname{Re}\left[\breve{G}_{\perp}^{\mathrm{TEM}}\left(0, \omega_{\mathrm{cav}}\right)\right]-\mu \frac{\omega_{0}}{\omega_{\mathrm{cav}}} \sum_{\mathbf{g}} \frac{\omega_{\mathrm{cav}}^{2} \xi^{2} e^{-\eta^{2}|\mathbf{K}-\mathbf{g}|^{2}}}{\omega_{\mathbf{K}-\mathbf{g}}^{2}-\omega_{\mathrm{cav}}^{2}}-\frac{\mu}{3} \frac{\omega_{0}}{\omega_{\mathrm{cav}}} \sum_{\mathbf{g}} \frac{\omega_{\mathrm{cav}}^{2} \xi^{2}}{\omega_{\mathbf{K}-\mathbf{g}}^{2}-\omega_{\mathrm{cav}}^{2}} \operatorname{Re}\left[e^{i \frac{\pi}{3} \delta} e^{-i \mathbf{g} \cdot\left(\mathbf{d}_{\mathrm{A}}-\mathbf{d}_{\mathrm{B}}\right)}\right. \\
& \left.+e^{-i \frac{\pi}{3} \delta} e^{-i \mathbf{g} \cdot\left(\mathbf{d}_{\mathrm{A}}-\mathbf{d}_{\mathrm{C}}\right)}-e^{-i \frac{2 \pi}{3} \delta} e^{-i \mathbf{g} \cdot\left(\mathbf{d}_{\mathrm{B}}-\mathbf{d}_{\mathrm{C}}\right)}\right] .
\end{aligned}
$$

Note we have used the regularization procedure to numerically evaluate the difference between the first two divergent terms in Eq. (B9), but we have neglected the Gaussian cut-off for the intersublattice terms because it has a negligible effect on the interactions between dipoles. Similarly, the Dirac velocity can be decomposed as $v_{\mathrm{D}}=v_{\|}+v_{\perp}$, where the contribution from the Coulomb interactions is

$$
\begin{aligned}
v_{\|}= & \frac{\mu}{\sqrt{3}} \frac{\omega_{0}}{\omega_{\text {cav }}} \sum_{\mathbf{R}} \operatorname{Re}\left[i\left(\mathbf{R}+\mathbf{d}_{\mathrm{A}}-\mathbf{d}_{\mathrm{C}}\right)_{y} G_{\|}\left(\mathbf{R}+\mathbf{d}_{\mathrm{A}}-\mathbf{d}_{\mathrm{C}}\right) e^{-i \frac{\pi}{3} \delta} e^{-i \mathbf{K} \cdot\left(\mathbf{R}+\mathbf{d}_{\mathrm{A}}-\mathbf{d}_{\mathrm{C}}\right)}\right. \\
& \left.-i\left(\mathbf{R}+\mathbf{d}_{\mathrm{A}}-\mathbf{d}_{\mathrm{B}}\right)_{y} G_{\|}\left(\mathbf{R}+\mathbf{d}_{\mathrm{A}}-\mathbf{d}_{\mathrm{B}}\right) e^{i \frac{\pi}{3} \delta} e^{-i \mathbf{K} \cdot\left(\mathbf{R}+\mathbf{d}_{\mathrm{A}}-\mathbf{d}_{\mathrm{B}}\right)}\right]
\end{aligned}
$$

and the contribution from the photon-mediated interactions reads

$$
\begin{aligned}
v_{\perp}= & \frac{\mu}{\sqrt{3}} \frac{\omega_{0}}{\omega_{\mathrm{cav}}} \sum_{\mathbf{g}} \frac{2 c^{2} \omega_{\mathrm{cav}}^{2} \xi^{2}}{\left(\omega_{\mathbf{K}-\mathbf{g}}^{2}-\omega_{\mathrm{cav}}^{2}\right)^{2}}(\mathbf{K}-\mathbf{g})_{y} \\
& \times \operatorname{Re}\left[e^{-i \frac{\pi}{3} \delta} e^{-i \mathbf{g} \cdot\left(\mathbf{d}_{\mathrm{A}}-\mathbf{d}_{\mathrm{C}}\right)}-e^{i \frac{\pi}{3} \delta} e^{-i \mathbf{g} \cdot\left(\mathbf{d}_{\mathrm{A}}-\mathbf{d}_{\mathrm{B}}\right)}\right] .
\end{aligned}
$$

Finally, the Dirac mass is given by Eq. (23) and Eq. (24).

\section{APPENDIX C: COULOMB AND PHOTON-MEDIATED INTERACTIONS FOR LARGE CAVITY WIDTHS}

Here we show that the essential physics for large cavity widths is dominated by the Coulomb interactions. In Fig. 2(a) and Fig. 4(b) we neglected the weak photonmediated interactions for clarity. In Fig. 5(a) we show the polariton dispersion for the shrunken metasurface $(\delta=0.05)$ 
(a)

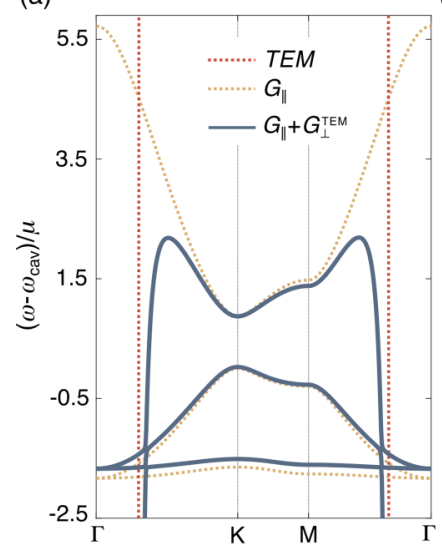

(b)

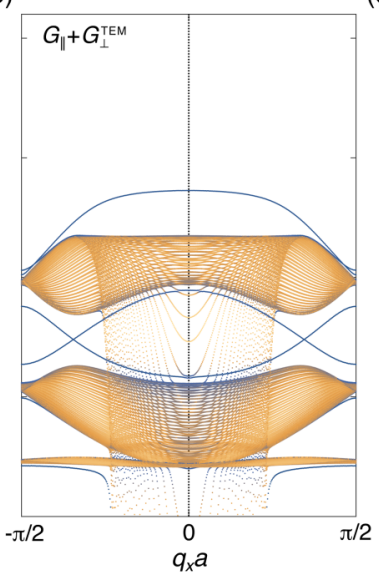

(c)

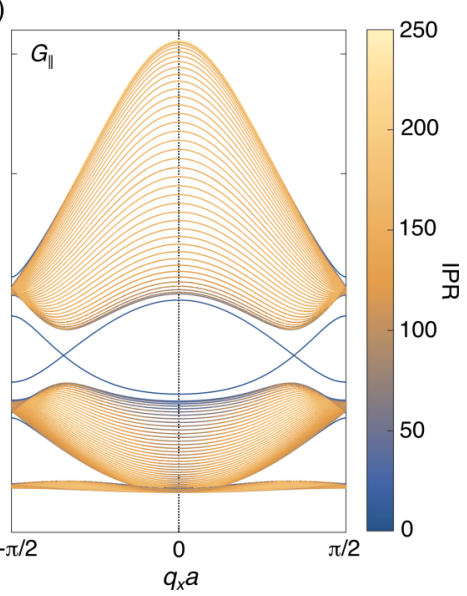

FIG. 5. (a) Polariton dispersion for the shrunken metasurface with $L=5 a$ where we include the full dipole-dipole interactions (solid blue line). We also show the linear dispersion of the TEM cavity mode (dashed red line) and the approximate quasistatic dispersion where we include only the Coulomb interactions (dashed orange line). (b) Polariton dispersion for the interface with $L=5 a$ where we include the full dipole-dipole interactions. The color of the bands represents the IPR, where blue/yellow bands correspond to localized/extended states. (c) Same as panel (b) but including only the Coulomb interactions. One observes that the dispersion of the valley-Hall edge states is only slightly modified by the weak photon-mediated interactions. Results obtained with $\delta=0.05, a=0.1 \lambda_{0}$ and $\mu=0.001 \omega_{0}$.

with a large cavity width where we include the full dipoledipole interactions (solid blue line). For comparison, we also plot the linear dispersion of the TEM cavity mode (dashed red line) and the approximate quasistatic dispersion where we include only the dominant Coulomb interactions (dashed orange line). The light-matter interaction results in a characteristic anticrossing between the bright quasistatic mode and the TEM cavity mode, but there is very little effect from the weak photon-mediated interactions near the $\mathrm{K} / \mathrm{K}^{\prime}$ points which is where our focus is. However, the hybridization results in polariton states that cross the quasistatic band gap which are predominantly of photonic character.

In Fig. 5(b) we show the polariton dispersion for the interface with a large cavity width where we include the full dipole-dipole interactions, and we compare this with (a)

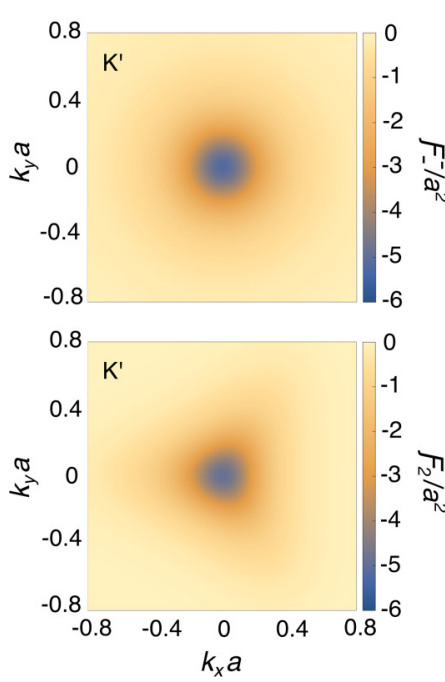

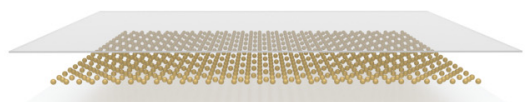
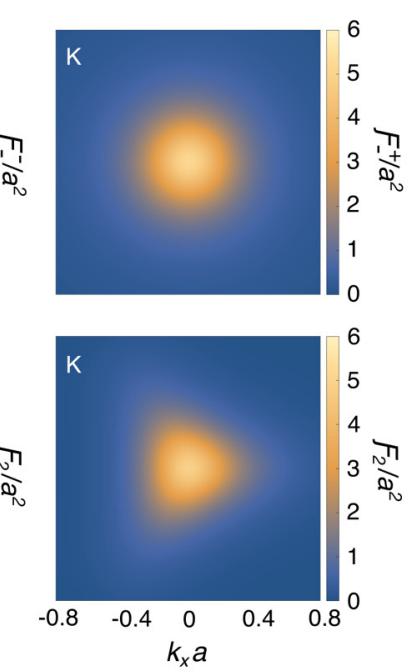

(b)
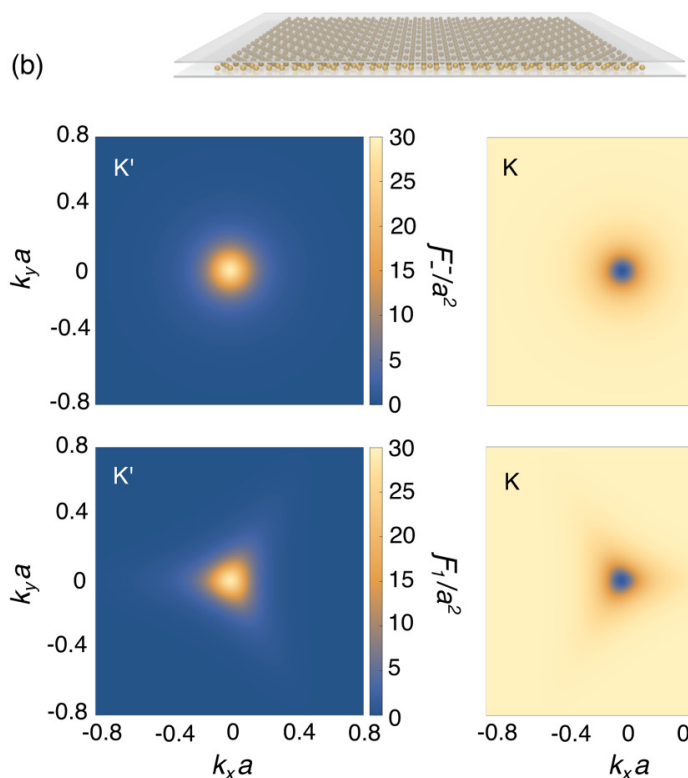
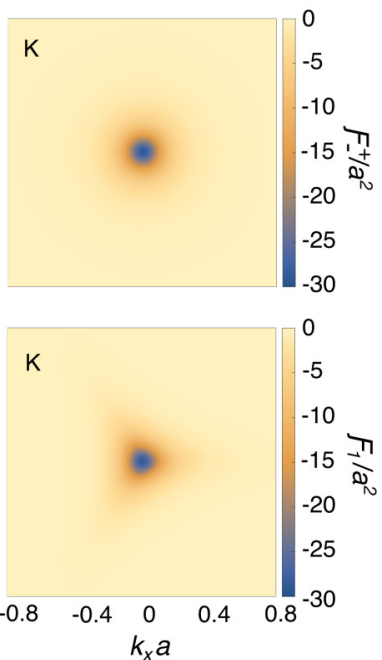

FIG. 6. (a) Berry curvature $(\lambda=-)$ near the $\mathrm{K} / \mathrm{K}^{\prime}$ points (right/left panel) for the shrunken metasurface with $L=5 a$. The top panels are obtained from the effective Dirac Hamiltonian and the bottom panels are obtained from the dynamical matrix. (b) Same as panel (a) but with $L=0.5 a$. The full Berry curvature is trigonally warped and exhibits $C_{3}$ symmetry about the $\mathrm{K} / \mathrm{K}^{\prime}$ points, while the effective Berry curvature is isotropic. Apart from this small difference, the Dirac Hamiltonian captures the essential Berry curvature near the $\mathrm{K} / \mathrm{K}^{\prime}$ points. Results obtained with $\delta=0.05, a=0.1 \lambda_{0}$ and $\mu=0.001 \omega_{0}$. 
the approximate quasistatic dispersion in Fig. 5(c) where we include only the dominant Coulomb interactions. The color of the bands represents the inverse participation ratio IPR = $\sum_{j}\left|p_{j}\right|^{4} /\left(\sum_{j}\left|p_{j}\right|\right)^{2}$, where the blue/yellow color represents localized/extended states. Note that because we apply periodic boundary conditions in the $y$ direction, the dispersion plots also contain the valley-Hall edge states located on the $\mathrm{B} / \mathrm{A}$ interface as well as the A/B interface. Due to the absence of a complete band gap, the bulk photonlike states overlap part of the edge state spectrum (blue bands). However, one observes that the edge states only couple weakly to these bulk states (as evidenced by the very small anticrossings), and thus the weak photon-mediated interactions have very little effect on the dispersion of the valley-Hall edge states for this regime of parameters. We should emphasize that the numerical simulations in Fig. 4(d) do include the weak photon-mediated interactions; this demonstrates that the valley-Hall edge states can be routed around sharp corners without any significant scattering into the bulk, despite the absence of a complete band gap.

\section{APPENDIX D: BERRY CURVATURE NEAR THE K/K' POINTS}

Here we show that Eq. (25) captures the essential Berry curvature near the $\mathrm{K} / \mathrm{K}^{\prime}$ points in the two limiting interaction regimes. We can calculate the full Berry curvature from the dynamical matrix which, for the $n$th band, reads

$$
\begin{aligned}
\mathcal{F}_{n}(\mathbf{q})= & i \sum_{m \neq n} \frac{\left\langle\varphi_{n}(\mathbf{q})\left|\frac{\partial}{\partial q_{x}} \mathcal{D}(\mathbf{q})\right| \varphi_{m}(\mathbf{q})\right\rangle\left\langle\varphi_{m}(\mathbf{q})\left|\frac{\partial}{\partial q_{y}} \mathcal{D}(\mathbf{q})\right| \varphi_{n}(\mathbf{q})\right\rangle}{\left[\check{\alpha}_{m}^{-1}(\mathbf{q})-\check{\alpha}_{n}^{-1}(\mathbf{q})\right]^{2}} \\
& + \text { c.c. . }
\end{aligned}
$$

Here $\left|\varphi_{m}\right\rangle$ and $\check{\alpha}_{m}^{-1}$ are the eigenvectors and eigenvalues of the dynamical matrix for the $m$ th band, respectively, where we evaluate the dynamical matrix and polarizability correction at the cavity resonant frequency. Note, while the effective Berry curvature within the two-band model is only accurate when the other band is well separated in frequency, Eq. (D1) applies to any band and is valid for all cavity widths. Furthermore, Eq. (D1) is valid for any perturbation while Eq. (25) is only accurate for small perturbations.

In Fig. 6(a) we compare the Berry curvature $(\lambda=-)$ calculated from the effective Dirac Hamiltonian (top panels) and the dynamical matrix (bottom panels) for the shrunken metasurface $(\delta=0.05)$ with a large cavity width. Figure $6(\mathrm{~b})$ is the same as Fig. 6(a) but for a small cavity width. The full Berry curvature is trigonally warped and exhibits $C_{3}$ symmetry about the $\mathrm{K} / \mathrm{K}^{\prime}$ points; this feature is missed by the effective Dirac Hamiltonian where the corresponding Berry curvature is isotropic. However, apart from this slight difference, the Berry curvature peaks have very similar magnitudes and therefore the effective Dirac Hamiltonian evidently captures the essential Berry curvature near the $\mathrm{K} / \mathrm{K}^{\prime}$ points in both limiting interaction regimes for small perturbations.
[1] L. Lu, J. D. Joannopoulos, and M. Soljačić, Topological photonics, Nat. Photonics 8, 821 (2014).

[2] A. B. Khanikaev and G. Shvets, Two-dimensional topological photonics, Nat. Photonics 11, 763 (2017).

[3] T. Ozawa, H. M. Price, A. Amo, N. Goldman, M. Hafezi, L. Lu, M. C. Rechtsman, D. Schuster, J. Simon, O. Zilberberg, and I. Carusotto, Topological photonics, Rev. Mod. Phys. 91, 015006 (2019).

[4] F. D. M. Haldane and S. Raghu, Possible Realization of Directional Optical Waveguides in Photonic Crystals with Broken Time-Reversal Symmetry, Phys. Rev. Lett. 100, 013904 (2008).

[5] Z. Wang, Y. Chong, J. D. Joannopoulos, and M. Soljačić, Observation of unidirectional backscattering-immune topological electromagnetic states, Nature (London) 461, 772 (2009).

[6] T. Karzig, C.-E. Bardyn, N. H. Lindner, and G. Refael, Topological Polaritons, Phys. Rev. X 5, 031001 (2015).

[7] S. Klembt, T. H. Harder, O. A. Egorov, K. Winkler, R. Ge, M. A. Bandres, M. Emmerling, L. Worschech, T. C. H. Liew, M. Segev, C. Schneider, and S. Höfling, Exciton-polariton topological insulator, Nature (London) 562, 552 (2018).

[8] M. Hafezi, E. A. Demler, M. D. Lukin, and J. M. Taylor, Robust optical delay lines with topological protection, Nat. Phys. 7, 907 (2011).

[9] M. Hafezi, S. Mittal, J. Fan, A. Migdall, and J. M. Taylor, Imaging topological edge states in silicon photonics, Nat. Photonics 7, 1001 (2013).
[10] K. Fang, Z. Yu, and S. Fan, Realizing effective magnetic field for photons by controlling the phase of dynamic modulation, Nat. Photonincs 6, 782 (2012).

[11] A. B. Khanikaev, S. H. Mousavi, W.-K. Tse, M. Kargarian, A. H. MacDonald, and G. Shvets, Photonic topological insulators, Nat. Mater. 12, 233 (2013).

[12] X. Cheng, C. Jouvaud, X. Ni, S. H. Mousavi, A. Z. Genack, and A. B. Khanikaev, Robust reconfigurable electromagnetic pathways within a photonic topological insulator, Nat. Mater. 15, 542 (2016).

[13] M. C. Rechtsman, J. M. Zeuner, Y. Plotnik, Y. Lumer, D. Podolsky, F. Dreisow, S. Nolte, M. Segev, and A. Szameit, Photonic Floquet topological insulators, Nature (London) 496, 196 (2013).

[14] L.-H. Wu and X. Hu, Scheme for Achieving a Topological Photonic Crystal by Using Dielectric Material, Phys. Rev. Lett. 114, 223901 (2015).

[15] T. Ma and G. Shvets, All-Si valley-Hall photonic topological insulator, New J. Phys. 18, 025012 (2016).

[16] X. Wu, Y. Meng, J. Tian, Y. Huang, H. Xiang, D. Han, and W. Wen, Direct observation of valley-polarized topological edge states in designer surface plasmon crystals, Nat. Commun. 8, 1304 (2017).

[17] Z. Gao, Z. Yang, F. Gao, H. Xue, Y. Yang, J. Dong, and B. Zhang, Valley surface-wave photonic crystal and its bulk/edge transport, Phys. Rev. B 96, 201402(R) (2017). 
[18] F. Gao, H. Xue, Z. Yang, K. Lai, Y. Yu, X. Lin, Y. Chong, G. Shvets, and B. Zhang, Topologically-protected refraction of robust kink states in valley photonic crystals, Nat. Phys. 14, 140 (2018).

[19] Y. Zeng, U. Chattopadhyay, B. Zhu, B. Qiang, J. Li, Y. Jin, L. Li, A. G. Davies, E. H. Linfield, B. Zhang, Y. Chong, and Q. J. Wang, Electrically pumped topological laser with valley edge modes, Nature (London) 578, 246 (2020).

[20] Y. Yang, Y. Yamagami, X. Yu, P. Pitchappa, B. Zhang, M. Fujita, T. Nagatsuma, and R. Singh, Terahertz topological photonics for on-chip communication, Nat. Photonics 14, 446 (2020).

[21] J. Noh, S. Huang, K. P. Chen, and M. C. Rechtsman, Observation of Photonic Topological Valley Hall Edge States, Phys. Rev. Lett. 120, 063902 (2018).

[22] M. I. Shalaev, W. Walasik, A. Tsukernik, Y. Xu, and N. M. Litchinitser, Robust topologically protected transport in photonic crystals at telecommunication wavelengths, Nat. Nanotechnol. 14, 31 (2019).

[23] X.-T. He, E.-T. Liang, J.-J. Yuan, H.-Y. Qiu, X.-D. Chen, F.-L. Zhao, and J.-W. Dong, A silicon-on-insulator slab for topological valley transport, Nat. Commun. 10, 872 (2019).

[24] M. J. Mehrabad, A. P. Foster, R. Dost, A. M. Fox, M. S. Skolnick, and L. R. Wilson, Chiral topological photonics with an embedded quantum emitter, Optica 7, 1690 (2020).

[25] S. Barik, A. Karasahin, S. Mittal, E. Waks, and M. Hafezi, Chiral quantum optics using a topological resonator, Phys. Rev. B 101, 205303 (2020).

[26] I. Martin, Y. M. Blanter, and A. F. Morpurgo, Topological Confinement in Bilayer Graphene, Phys. Rev. Lett. 100, 036804 (2008).

[27] G. V. Semenoff, V. Semenoff, and F. Zhou, Domain Walls in Gapped Graphene, Phys. Rev. Lett. 101, 087204 (2008).

[28] J. Li, A. F. Morpurgo, M. Buttiker, and I. Martin, Marginality of bulk-edge correspondence for single-valley Hamiltonians, Phys. Rev. B 82, 245404 (2010).

[29] J. Jung, F. Zhang, Z. Qiao, and A. H. MacDonald, Valley-Hall kink and edge states in multilayer graphene, Phys. Rev. B 84, 075418 (2011).

[30] F. Zhang, A. H. MacDonald, and E. J. Mele, Valley Chern numbers and boundary modes in gapped bilayer graphene, Proc. Natl. Acad. Sci. USA 110, 10546 (2013).

[31] A. Vaezi, Y. Liang, D. H. Ngai, L. Yang, and E.-A. Kim, Topological Edge States at a Tilt Boundary in Gated Multilayer Graphene, Phys. Rev. X 3, 021018 (2013).

[32] L. Ju, Z. Shi, N. Nair, Y. Lv, C. Jin, J. Velasco, Jr., C. OjedaAristizabal, H. A. Bechtel, M. C. Martin, A. Zettl, J. Analytis, and F. Wang, Topological valley transport at bilayer graphene domain walls, Nature 520, 650 (2015).

[33] J. Li, K. Wang, K. McFaul, Z. Zern, Y. Ren, K. Watanabe, T. Taniguchi, Z. Qiao, and J. Zhu, Gate-controlled topological conducting channels in bilayer graphene, Nat. Nanotech. 11, 1060 (2016).

[34] C.-R. Mann, T. J. Sturges, G. Weick, W. L. Barnes, and E. Mariani, Manipulating type-I and type-II Dirac polaritons in cavity-embedded honeycomb metasurfaces, Nat. Commun. 9, 2194 (2018).

[35] C.-R. Mann, S. A. R. Horsley, and E. Mariani, Tunable pseudomagnetic fields for polaritons in strained metasurfaces, Nat. Photonics 14, 669 (2020).
[36] G. Weick, C. Woollacott, W. L. Barnes, O. Hess, and E. Mariani, Dirac-Like Plasmons in Honeycomb Lattices of Metallic Nanoparticles, Phys. Rev. Lett. 110, 106801 (2013).

[37] S. Lamowski, C.-R. Mann, F. Hellbach, E. Mariani, G. Weick, and F. Pauly, Plasmon polaritons in cubic lattices of spherical metallic nanoparticles, Phys. Rev. B 97, 125409 (2018).

[38] C. A. Downing, T. J. Sturges, G. Weick, M. Stobińska, and L. Martín-Moreno, Topological Phases of Polaritons in a Cavity Waveguide, Phys. Rev. Lett. 123, 217401 (2019).

[39] F. Fernique, and G. Weick, Plasmons in two-dimensional lattices of near-field coupled nanoparticles, Phys. Rev. B 102 045420 (2020).

[40] M. Proctor, P. A. Huidobro, S. A. Maier, R. V. Craster, and M. P. Makwana, Manipulating topological valley modes in plasmonic metasurfaces, Nanophotonics 9, 2019 (2020).

[41] J. Yuen-Zhou, S. S. Saikin, N. Y. Yao, and A. Aspuru-Guzik, Topologically protected excitons in porphyrin thin films, Nat. Mater. 13, 1026 (2014).

[42] J. Yuen-Zhou, S. K. Saikin, T. Zhu, M. C. Onbasli, C. A. Ross, V. Bulovic, and M. A. Baldo, Plexciton Dirac points and topological modes, Nat. Commun. 7, 11783 (2016).

[43] A. González-Tudela, C.-L. Hung, D. E. Chang, J. I. Cirac, and H. J. Kimble, Subwavelength vacuum lattices and atomatom interactions in two-dimensional photonic crystals, Nat. Photonics 9, 320 (2015).

[44] D. Barredo, S. de Léséleuc, V. Lienhard, T. Lahaye, and A. Browaeys, An atom-by-atom assembler of defect-free arbitrary two-dimensional atomic arrays, Science 354, 1021 (2016).

[45] S. de Léséleuc, V. Lienhard, P. Scholl, D. Barredo, S. Weber, N. Lang, H. P. Büchler, T. Lahaye, and A. Browaeys, Observation of a symmetry-protected topological phase of interacting bosons with Rydberg atoms, Science 365, 775 (2019).

[46] R. J. Bettles, S. A. Gardiner, and C. S. Adams, Enhanced Optical Cross Section Via Collective Coupling of Atomic Dipoles in a 2D Array, Phys. Rev. Lett. 116, 103602 (2016).

[47] E. Shahmoon, D. S. Wild, M. D. Lukin, and S. F. Yelin, Cooperative Resonances in Light Scattering from Two-Dimensional Atomic Arrays, Phys. Rev. Lett. 118, 113601 (2017).

[48] R. J. Bettles, J. Minář, C. S. Adams, I. Lesanovsky, and B. Olmos, Topological properties of a dense atomic lattice gas, Phys. Rev. A 96, 041603(R) (2017).

[49] J. Perczel, J. Borregaard, D. E. Chang, H. Pichler, S. F. Yelin, P. Zoller, and M. D. Lukin, Topological Quantum Optics in Two-Dimensional Atomic Arrays, Phys. Rev. Lett. 119, 023603 (2017).

[50] R. Bekenstein, I. Pikovski, H. Pichler, E. Shahmoon, S. F. Yelin, and M. D. Lukin, Quantum metasurfaces with atom arrays, Nat. Phys. 16, 676 (2020).

[51] A. A. Svidzinsky, J.-T. Chang, and M. O. Scully, Cooperative spontaneous emission of $\mathrm{N}$ atoms: Many-body eigenstates, the effect of virtual Lamb shift processes and analogy with radiation of $\mathrm{N}$ classical oscillators, Phys. Rev. A 81, 053821 (2010).

[52] J. Lu, C. Qiu, S. Xu, Y. Ye, M. Ke, and Z. Liu, Dirac cones in two-dimensional artificial crystals for classical waves, Phys. Rev. B 89, 134302 (2014).

[53] X. Ni, M. A. Gorlach, A. Alù, and A. B. Khanikaev, Topological edge states in acoustic Kagome lattices, New J. Phys. 19, 055002 (2017). 
[54] Z. Zhang, Y. Tian, Y. Wang, S. Gao, Y. Cheng, X. Liu, and J. Christensen, Directional acoustic antennas based on valley-Hall topological insulators, Adv. Mater. 30, 1803229 (2018).

[55] H. Xue, Y. Yang, F. Gao, Y. Chong, and B. Zhang, Acoustic higher-order topological insulator on a kagome lattice, Nat. Mater. 18, 108 (2019).

[56] X. Ni, M. Weiner, A. Alù, and A. B. Khanikaev, Observation of higher-order topological acoustic states protected by generalized chiral symmetry, Nat. Mater. 18, 113 (2019).

[57] N. Lera, D. Torrent, P. San-Jose, J. Christensen, and J. V. Alvarez, Valley Hall phases in kagome lattices, Phys. Rev. B 99, 134102 (2019).

[58] S. Wong, M. Saba, O. Hess, and S. S. Oh, Gapless unidirectional photonic transport using all-dielectric kagome lattices, Phys. Rev. Research 2, 012011(R) (2020).
[59] M. Li, D. Zhirihin, M. Gorlach, X. Ni, D. Filonov, A. Slobozhanyuk, A. Alù, and A. B. Khanikaev, Higher-order topological states in photonic kagome crystals with long-range interactions, Nat. Photonics 14, 89 (2020).

[60] C.-R. Mann, Tunable Dirac polaritons in cavity-embedded metasurfaces, $\mathrm{PhD}$. thesis, University of Exeter, 2021, http://hdl.handle.net/10871/126986.

[61] A. Y. Nikitin, F. Guinea, F. J. García-Vidal, and L. MartínMoreno, Fields radiated by a nanoemitter in a graphene sheet, Phys. Rev. B 84, 195446 (2011).

[62] J. Perczel, J. Borregaard, D. E. Chang, S. F. Yelin, and M. D. Lukin, Topological Quantum Optics Using Atom-Like Emitter Arrays Coupled to Photonic Crystals, Phys. Rev. Lett. 124, 083603 (2020). 